GUTPA/95/01/1

\title{
Could There Be a Fourth Generation of Quarks Without More Leptons?
}

\author{
C. D. Froggatt ${ }^{1}$, D. J. Smith ${ }^{1}$ and H. B. Nielsen ${ }^{2}$ \\ ${ }^{1}$ Department of Physics and Astronomy, University of Glasgow, \\ Glasgow G12 8QQ, UK \\ 2 Niels Bohr Institute, Blegdamsvej 17-21, \\ DK 2100 Copenhagen, Denmark
}

\begin{abstract}
We investigate the possibility of adding a fourth generation of quarks. We also extend the Standard Model gauge group by adding another $S U(N)$ component. In order to cancel the contributions of the fourth generation of quarks to the gauge anomalies we must add a generation of fermions coupling to the $S U(N)$ group. This model has many features similar to the Standard Model and, for example, includes a natural generalisation of the Standard Model charge quantisation rule. We discuss the phenomenology of this model and, in particular, show that the infrared quasi-fixed point values of the Yukawa coupling constants put upper limits on the new quark masses close to the present experimental lower bounds.
\end{abstract}




\section{Introduction}

The LEP determinations of the invisible partial decay width of the $Z^{0}$ gauge boson show there are just three neutrinos of the usual type with mass less than $M_{Z} / 2$. This result is naturally interpreted to imply that there are just three generations of quarks and leptons. In this letter we consider the possibility that there is an extra generation of quarks without accompanying leptons. There is of course a well-known problem with such a "half generation" consisting only of quarks: the gauge anomalies associated with triangle diagrams, having electroweak gauge bosons (but no QCD gluons) attached to the vertices, would not cancel. So some further particles would have to be added to the model in order to cancel these anomalies. At first one could worry that it might be difficult to cancel all the anomalies in a simple way. However we shall argue that the uncancelled quark anomalies make up a natural unit that is rather easily compensated by a kind of techniquark, or rather technilepton since it does not couple to $S U(3)_{C}$, generation. Our philosophy in the present article is to seek extensions of the Standard Model (SM) incorporating a fourth quark generation and retaining as many of the distinctive properties of the SM gauge group as possible. We should even like to argue that the proposed fourth quark generation model is the unique extension of the SM, which retains most of the features of the SM and is still (just) viable phenomenologically.

So we now summarise the characteristic properties of the Standard Model Group $(\mathrm{SMG})$. SMG is defined to be the group $U(1) \otimes S U(2) \otimes S U(3)$ supplemented by the charge quantisation rule:

$$
\frac{y}{2}+\frac{1}{2} d+\frac{1}{3} t \equiv 0 \quad(\bmod 1)
$$

The duality $d$ has value 1 if the representation is an $S U(2)$ doublet and 0 if it is an $S U(2)$ singlet. The triality $t$ has value 1 if the representation is an $S U(3)$ triplet, 0 if it is an $S U(3)$ singlet, and -1 if it is an $S U(3)$ anti-triplet. The imposition of the charge quantisation rule eq. (1) corresponds to dividing out an appropriate discrete group D:

$$
S M G \equiv U(1) \otimes S U(2) \otimes S U(3) / D
$$

In fact $\mathrm{SMG}$ is the proper subgroup [1, 2] $S(U(2) \otimes U(3))$ of $S U(5)$. The SM charge quantisation rule eq. (1) implies that the value of the weak hy- 
percharge $y / 2$ of an irreducible representation of SMG determines uniquely the duality and triality of the representation.

We wish to consider extensions of SMG with the same distinctive features as SMG itself and are thereby led to add another $S U(N)$ factor to the sequence $U(1) \otimes S U(2) \otimes S U(3)$, giving gauge groups of the type:

$$
S M G_{2,3, N} \equiv U(1) \otimes S U(2) \otimes S U(3) \otimes S U(N) / D_{N}
$$

Here $D_{N}$ is a discrete group which ensures that a generalised charge quantisation rule of the form:

$$
\frac{y}{2}+\frac{1}{2} d+\frac{1}{3} t+\frac{m_{N}}{N} n \equiv 0 \quad(\bmod 1)
$$

is satisfied, where $n$ is the $\mathrm{N}$-ality of the representation and $m_{N}$ is an integer which is not a multiple of $\mathrm{N}$.

Another feature that we take over from the SM is the principle of using only small (fundamental or singlet) fermion representations; a feature not shared with supersymmetric models, where the gauginos are in adjoint representations. We note that $\mathrm{N}$-ality has value 1 if a representation is an $S U(N)$ N-plet, 0 if it is an $S U(N)$ singlet, and -1 if it is an $S U(N)$ anti-Nplet. In order that the generalised charge quantisation rule, eq. (丰), continues to determine the duality, triality and $\mathrm{N}$-ality of an irreducible representation once $y / 2$ is specified, 2,3 and $\mathrm{N}$ must be mutually prime.

We will consider the fundamental scale to be the Planck mass $\left(M_{\text {Planck }}\right)$ and our models will be a full description of physics without gravity below this scale. By requiring an anomaly free theory and the absence of any Landau poles below $M_{\text {Planck }}$, we obtain an essentially unique extension of the SM with an extra generation of quarks, but with the extra generation of leptons replaced by fermions in the fundamental representations of the $S U(N)$ component of $S M G_{2,3, N}$.

We assume that the extra $S U(N)$ component of the gauge group is not spontaneously broken and therefore confines forming fermion condensates. As we already know from the SM, the $S U(3)$ component acts as a technicolour group [3] and gives a contribution to the $W^{ \pm}$and $Z^{0}$ masses. In the SM this contribution is very small but when confining groups with $N>3$ are considered we must carefully consider the effect this will have. Since we are not wanting the complications of extended technicolour in order to generate 
quark and lepton masses, we assume that there is a Higgs doublet and that the masses of the weak gauge bosons are generated by a combination of the Higgs sector of the theory and the technicolour effects of the gauge groups. Thus the extra $S U(N)$ component of the gauge group acts only as a partial technicolour $\llbracket$. It follows that the $S U(N)$ confinement scale must be at the $\mathrm{TeV}$ scale or below.

In order to avoid conflict with precision electroweak data, it is not phenomenologically consistent to introduce a large number of extra $S U(2)_{L}$ doublets. We shall therefore concentrate on the phenomenology of the $S M G_{2,3,5}$ model which has the minimal value of $N=5$.

\section{Structure of the $S M G_{2,3, N}$ Model}

The three SM generations form representations of $S M G_{2,3, N}$ and we now wish to consider a new "generation" of fermions coupling to the $S U(N)$, but not the $S U(3)$, component of the group. We assume all the fermions get a mass via the SM Higgs mechanism and, by analogy with the SM quarks, we consider a generation of $S U(N)$ "quarks" made up of the left-handed fermion representation $(y, 2, N)$ together with the left-handed anti-fermion representations $(-[y+1], 1, \bar{N})$ and $(-[y-1], 1, \bar{N})$. The weak hypercharge $y$ must of course satisfy the charge quantisation rule eq.(国). We now consider the cancellation of the anomalies generated by such a generation of $S U(N)$ "quarks".

The contributions to each type of anomaly from this generation of $S U(N)$ "quarks" are:

$$
\begin{aligned}
& {[S U(N)]^{3} \quad \rightarrow \quad 0} \\
& {[S U(N)]^{2} U(1) \rightarrow 2 y-(y+1)-(y-1) \quad=0} \\
& {[\text { Grav }]^{2} U(1) \rightarrow 2 y-(y+1)-(y-1) \quad=0} \\
& {[U(1)]^{3} \quad \rightarrow \quad N\left[2 y^{3}-(y+1)^{3}-(y-1)^{3}\right]=-6 N y} \\
& {[S U(2)]^{2} U(1) \rightarrow \quad N y}
\end{aligned}
$$

So we must cancel the resulting $[S U(2)]^{2} U(1)$ and $[U(1)]^{3}$ anomalies against other fermion representations in order to obtain a consistent theory. We can 
in fact cancel the anomalies against a fourth generation of $S U(3)_{C}$ quarks, in just the same way that the quark anomalies cancel the lepton anomalies in the three SM generations 1 .

The non-zero anomalies due to a fourth generation of quarks are:

$$
\begin{array}{lll}
{[U(1)]^{3}} & \rightarrow 3\left[2\left(\frac{1}{3}\right)^{3}-\left(\frac{4}{3}\right)^{3}+\left(\frac{2}{3}\right)^{3}\right] & =-6 \\
{[S U(2)]^{2} U(1)} & \rightarrow 3 \cdot \frac{1}{3} & =1
\end{array}
$$

Anomaly cancellation between a generation of $S U(N)$ "quarks" and a fourth generation of $S U(3)_{C}$ quarks gives the condition:

$$
N y+1=0
$$

and hence

$$
y=-\frac{1}{N}
$$

However the weak hypercharge $y$ of the $S U(N)$ "quarks" must satisfy the charge quantisation rule eq.(11), which takes the form:

$$
y=2 J-1-\frac{2 m_{N}}{N}
$$

where $J$ is an integer. So we have a solution with $J=1$ and

$$
m_{N}=\frac{1}{2}(N+1)
$$

Table 1 shows the properties of the left-handed fermions belonging to such a generation of $S U(N)$ "quarks" for the phenomenologically interesting case: $\mathrm{N}=5$. Finally we remark that $N$ is odd and hence Table 1 contains an odd number of $S U(2)$ doublets. Combined with the three $S U(2)$ doublets in a fourth generation of quarks, this gives an even number of doublets and hence there is no Witten discrete $S U(2)$ anomaly [5].

\footnotetext{
${ }^{1}$ The resulting generation of $S U(3)$ quarks and $S U(N)$ "quarks" is the smallest massprotected anomaly free representation which couples non-trivially to all the components of the gauge group $S M G_{2,3, N}$. In the technicolour literature these $S U(N)$ "quarks" are usually called technileptons as they do not couple to $S U(3)_{C}$.
} 


\section{Phenomenology of the $S M G_{2,3,5}$ Model}

We shall now discuss the minimal extension of the SM in our class of models. It is based on the gauge group $S M G_{2,3,5} \equiv U(1) \otimes S U(2) \otimes S U(3) \otimes S U(5) / D_{5}$ and, in addition to the three SM generations, contains a fourth generation of quarks and a single generation of $S U(5)$ "quarks" as specified in Table 1 .

We assume that the $S U(5)$ component of the gauge group confines and that the $S U(5)$ "quarks" form condensates. These condensates have the same quantum numbers as the SM Higgs boson and contribute to the $W^{ \pm}$and $Z^{0}$ masses, via the usual technicolour [3] mechanism.

We stress that we are not proposing a technicolour model as such, but simply taking into account the unavoidable effect that adding an $S U(5)$ group has. We are assuming that the Higgs sector of our models is the same as in the SM, i.e. one Higgs doublet. Then the VEV due to the Higgs field, $<\phi_{W S}>$, is related to the total VEV, $v$, and the contribution from $S U(5)$ due to fermion condensates, $F_{\pi_{N}}$, by the relation

$$
<\phi_{W S}>^{2}+F_{\pi_{N}}^{2}=v^{2}=(246 \mathrm{GeV})^{2}
$$

which is exactly the same as in technicolour models with a scalar [4].

The fermion running masses, $m_{f}$, are related to the Higgs field VEV in the usual way;

$$
m_{f}=\frac{y_{f}}{\sqrt{2}}<\phi_{W S}>
$$

where $y_{f}$ is the Yukawa coupling constant for the fermion $f$ ( $y$ is used for both Yukawa coupling and weak hypercharge but it should be obvious from the context which is being referred to). In order to avoid any significant suppression of the top quark and other fermion masses, due to the reduction of $\left\langle\phi_{W S}\right\rangle$ below its SM value, we usually imagine taking

$$
F_{\pi_{N}} \approx 75 \mathrm{GeV}
$$

and thus

$$
<\phi_{W S}>\approx 234 \mathrm{GeV}
$$

This leads to a $5 \%$ reduction of the fermion masses relative to the value if there was no technicolour contribution. It also implies a typical $S U(5)$ "hadron" mass [6], due to confinement, of $\Lambda \simeq 400 \mathrm{GeV}$. 
We now briefly discuss the experimental constraints on the masses of the new fermions in the model. First we will consider the usual $S U(3)$ quarks and then the $S U(5)$ "quarks".

The top quark has recently been observed by the CDF and D0 [7] collaborations, with a mass of $180 \pm 12 \mathrm{GeV}$. We use the dilepton mode analyses of the CDF and D0 [8] groups to place a lower limit on the possible masses of a fourth generation of $t^{\prime}$ and $b^{\prime}$ quarks. If the $t^{\prime}$ quark is lighter than the $b^{\prime}$ quark, it is expected to decay via the mode $t^{\prime} \rightarrow b W^{+}$and hence give a dilepton signal similar to the top quark. If the $b^{\prime}$ quark is lighter than the $t^{\prime}$ and top quarks, it is expected to decay via the mode $b^{\prime} \rightarrow c W^{-}$and again give a similar dilepton signal. So we take the limit on the pole masses of possible fourth generation quarks, $t^{\prime}$ and $b^{\prime}$, to be

$$
M_{t^{\prime}}, M_{b^{\prime}}>130 \mathrm{GeV}
$$

from the dilepton analyses of the CDF and D0 groups 2 .

The above experimental limits do not apply to possible $S U(5)$ "quarks". These fermions would be more difficult to produce and detect at hadron colliders. As explained above, they would anyway be expected to be confined inside $S U(5)$ "hadrons" having masses of order $400 \mathrm{GeV}$. So we conclude that the $S U(5)$ "quarks" would be unlikely to be detected with current accelerators.

Precision electroweak data can be used to set limits on possible new physics at the electroweak scale. For example, the closeness of the observed value of the $\rho$ parameter $\left(\rho \equiv \frac{M_{W}^{2}}{M_{Z}^{2} \cos ^{2} \theta_{W}}\right)$ to unity [10] indicates that the mass squared differences within any new fermion $S U(2)$ doublets must be small $\left(\ll(100 \mathrm{GeV})^{2}\right)$. We shall assume that these mass splittings are small in our model. More generally any new physics which affects only the gauge boson self-energies can be parameterised by the $S, T$ and $U$ parameters [11 or some equivalent set of parameters [12, 13. The new physics contributions $T_{\text {new }}$ and $U_{\text {new }}$ to the $T$ and $U$ parameters in our model can be neglected.

The $S$ parameter provides a strong constraint on the number of new fermion doublets, since perturbatively a mass degenerate doublet contributes $\frac{1}{6 \pi}$ to $S$. Analysis of the precision electroweak data gives [14:

$$
S_{\text {new }}=-0.12 \pm 0.24
$$

\footnotetext{
${ }^{2}$ Different limits apply if other decay modes are dominant [9]
} 
for a Higgs mass $M_{H} \simeq 170 \mathrm{GeV}$. There are 8 new $S U(2)$ doublets in our $S M G_{2,3,5}$ model, made up of a fourth generation of quarks and a generation of $S U(5)$ "quarks". In a single SM generation, there are 4 doublets. So treating all the new fermions perturbatively, their contribution to $S_{\text {new }}$ is equivalent to that of two SM generations: $S_{n e w}=\frac{4}{3 \pi} \sim 0.42$. Thus, perturbatively, our model deviates by $\sim 2$ standard deviations from the experimental value of $S_{\text {new }}$. Non-perturbative corrections, estimated by analogy with QCD, tend to increase the $S U(5)$ fermion contribution to $S$ [15] by a factor $\sim 2$ and raise the deviation of our model from experiment to $\sim 3$ standard deviations. Our model is therefore just consistent with the precision electroweak data.

We now investigate the consistency of the model assuming it to be valid up to the Planck scale. In particular we require the absence of Landau poles below $M_{\text {Planck }}$.

Using the renormalisation group equations (RGEs), we first examine how the gauge coupling constants vary with energy in our model with 1 Higgs doublet, 3 SM generations, a fourth quark generation and a generation of $S U(5)$ "quarks". For convenience, we set the thresholds for all the unknown fermions (4th generation quarks and fermions coupling to $S U(5)$ ), as well as for the top quark and Higgs boson, to $M_{Z}$. The absence of Landau poles in this case will guarantee their absence if some of the thresholds are set higher than $M_{Z}$. There are four fine structure constants, which we shall label by $\alpha_{1}$, $\alpha_{2}, \alpha_{3}$ and $\alpha_{5}$, corresponding to the four gauge groups $U(1), S U(2), S U(3)$ and $S U(5)$ respectively. From [16] we find

$$
\begin{aligned}
& \alpha_{1}^{-1}\left(M_{Z}\right)=58.85 \pm 0.10 \\
& \alpha_{2}^{-1}\left(M_{Z}\right)=29.794 \pm 0.048 \\
& \alpha_{3}^{-1}\left(M_{Z}\right)=8.55 \pm 0.37
\end{aligned}
$$

where we have used the standard GUT normalisation for $\alpha_{1}$. For definiteness, we shall assume that $\alpha_{5}^{-1}\left(M_{Z}\right)=2$, although its precise value is unimportant. The first order RGEs [17, 18, 19 are easily integrated and give the results [20] shown in Fig. 11. We see that there are no problems with Landau poles below the Planck scale and our model is perturbatively consistent.

We can now use the RGEs to estimate upper limits on the values of the Yukawa couplings at the electroweak scale. This will lead to upper limits on the masses of the fermions. We do this by choosing initial values for the Yukawa couplings at the Planck scale and use the RGEs to see how they 
evolve, as they are run down to the electro-weak scale. Details of the RGEs we used in the approximation of no quark mixing are given in [20].

We have chosen the low energy scale to be $M_{Z}$. We observe fixed points similar to the case for the top quark in the SM [21]. However, the Yukawa coupling for any fermion at $M_{Z}$ depends on the Yukawa couplings of the other fermions. But there is an approximate infrared fixed point limit on $Y_{2}(S)=3 y_{t}^{2}+3 y_{t^{\prime}}^{2}+3 y_{b^{\prime}}^{2}+5 y_{5 u}^{2}+5 y_{5 d}^{2}$ where we have used $5 u$ and $5 d$ to label the $S U(5)$ "quarks" as a generalisation of the naming of the quarks. So one Yukawa coupling can be increased at the expense of the others. This limit is quite precise if there is only one strong interaction at low energies such as QCD in the SM 3 . We observe numerically that $Y_{2}(S) \approx 7.5 \pm 0.3$ provided the Yukawa couplings of the $t, t^{\prime}$ and $b^{\prime}$ quarks are greater than 1 , and that the Yukawa couplings of the $S U(5)$ "quarks" are less than the Yukawa couplings of the $t, t^{\prime}$ and $b^{\prime}$ quarks, at the Planck scale. The Yukawa couplings at the Planck scale in fig. 2 have been chosen so that $M_{t} \approx 170 \mathrm{GeV}$ and the fourth generation quark masses are above the current experimental limit of $130 \mathrm{GeV}$. Also $M_{b^{\prime}} \sim M_{t^{\prime}}$ and $M_{5 u} \sim M_{5 d}$ have been chosen so that there is only a small contribution to the $\rho$ parameter. Table 2 gives the value of the Yukawa couplings at $M_{Z}$ and the corresponding pole masses including the technicolour contribution to the VEV, as in eq. (10). The relation between the pole mass, $M_{f}$, and the running mass, $m_{f}$, of a fermion $f$ is given by:

$$
M_{f}=m_{f}\left(M_{f}\right)\left(1+\frac{4}{3} \alpha_{3}\left(M_{f}\right)\right)
$$

for the quarks, and for the $S U(5)$ "quarks" by:

$$
M_{f}=m_{f}\left(M_{f}\right)\left(1+\frac{12}{5} \alpha_{5}\left(M_{f}\right)\right)
$$

The masses in table 2 should be considered upper limits on the pole masses (the pole mass is identified with the experimentally measured quantity for the $t, t^{\prime}$ and $b^{\prime}$ quarks) of the fermions for this particular choice of Yukawa couplings at the Planck scale. For other choices of Yukawa couplings at the Planck scale we could, for example, increase the mass of the fourth generation of quarks but this would have to be compensated for by a reduction in the mass of some of the other fermions. These values for the masses are

\footnotetext{
${ }^{3}$ Detailed results for a general number of heavy SM generations are derived in 22 .
} 
consistent with current experimental limits but are not so high that all the new fermions could remain undetected for long. In fact the fourth generation quark masses may even be within the limits of current accelerators. However it is unlikely that the fermions coupling to $S U(5)$ could be observed; they would be confined inside $S U(5)$ "hadrons", with a confinement scale of order $400 \mathrm{GeV}$, and would have a small production cross section at hadron colliders. For this reason we consider the clearest evidence for this model would come from the detection of a fourth generation quark. The masses of some fermions could be increased, but not by much, since this would mean a reduction in the mass of other fermions. This means that this model is consistent and relatively easy to test.

We also note that in [20] we show that these fermion masses lead to a fixed point value of the Higgs self-coupling, which implies that the Higgs particle has a mass of approximately $170 \mathrm{GeV}$. This is the value we assumed for the Higgs mass when discussing the experimental limits on the $S$ parameter.

\section{Conclusion}

In answer to our title we would say that it is possible to have another generation of quarks without more leptons. However, we have shown that even with the smallest model of the types we proposed (taking the additional gauge group to be $S U(5))$, there are very severe bounds from both precision electroweak data and experimental lower limits on the fourth generation quark masses. We are clearly on the limit of agreement with precision electroweak data and this is sufficient to rule out the models with an additional $S U(N)$ where $N>5$ since there would then be even more $S U(2)$ doublets and so the $S$ parameter would deviate from experiment by more than 2-3 standard deviations. In the case of $N=5$ which we examined in detail we showed that it is possible for the fourth generation quarks to have masses above the current experimental limits. However, the upper limits from the infrared quasi-fixed point limits on the Yukawa couplings are not much above the experimental lower bounds. When we consider this together with the unnatural requirement (in the sense that it doesn't apply to the observed quarks) that the quark masses be approximately degenerate, it is clear that this model can only be consistent with a very limited choice of parameters. This is not enough to conclude that the model cannot be correct but more 
accurate measurements of the $S$ parameter and slightly higher limits on the masses of a fourth generation of quarks would certainly provide a definite conclusion.

\section{Acknowledgements}

We thank John Gunion, Hans Jensen, Victor Novikov, David Sutherland and Misha Vysotsky for helpful discussions. This work has been supported in part by INTAS Grant No. 93-3316 and PPARC Grant No. GR/J21231, the British Council, Cernfoelgeforskning and EF contract SC1 0340 (TSTS).

\section{References}

[1] C. D. Froggatt, H. B. Nielsen: Origin of Symmetries (World Scientific, Singapore, 1991).

[2] L. O'Raifeartaigh, Group Structure of Gauge Theories (Cambridge University Press, 1986).

[3] E. Fahri, L. Susskind: Phys. Rep. 74 (1981) 277.

[4] E. H. Simmons, Nucl. Phys. B 312 (1989) 253;

C.D. Carone, H. Georgi: Phys. Rev. D 49 (1994) 1427.

[5] E. Witten: Phys. Lett. B 117 (1982) 324.

[6] H. Georgi: Phys. Lett. B 298 (1993) 187.

[7] F. Abe et al.: Phys. Rev. Lett. 74 (1995) 2626;

S. Abachi et al.: Phys. Rev. Lett. 74 (1995) 2632.

[8] S. Abachi et al.: Phys. Rev. Lett. 72 (1994) 2138;

F. Abe et al.: Phys. Rev. D 50 (1994) 2966.

[9] J. F. Gunion, D. W. McKay, H. Pois: Phys. Lett. B 334 (1994) 339;

H. E. Haber: CERN-TH/95-178 (1995) hep-ph 9506426

[10] M. Veltman: Nucl. Phys. B 123 (1977) 89. 
[11] M. E. Peskin, T. Takeuchi: Phys. Rev. D 46 (1992) 381.

[12] G. Altarelli, R. Barbieri: Phys. Lett. B 253 (1991) 161.

[13] V. A. Novikov, L. B. Okun, M. I. Vysotsky: Nucl. Phys. B 397 (1993) 35 .

[14] J. Erler, P. Langacker: Phys. Rev. D 52 (1995) 441.

[15] K. Lane, Technicolor and Precision Tests of the Electroweak Interactions, in: Proc. of the 27th International Conference on High Energy Physics (Glasgow, 1994), eds. P. Bussey, I. Knowles: (IOP Publishing Ltd, 1995) p. 543.

[16] Particle Data Group: Phys. Rev. D 50 (1994) 1173.

[17] H. Georgi, H. Quinn, S. Weinberg: Phys. Rev. Lett. 33 (1974) 451.

[18] M. E. Machacek, M. T. Vaughn: Nucl. Phys. B 222 (1983) 83; Nucl. Phys. B 236 (1984) 221; Nucl. Phys. B 249 (1985) 70.

[19] H. Arason, D. J. Castaño, B. Kesthelyi, S. Mikaelian, E. J. Piard, P. Ramond, B. D. Wright: Phys. Rev. D 46 (1992) 3945.

[20] C. D. Froggatt, H. B. Nielsen, D. J. Smith: GUTPA-95-01-2 (1995).

[21] C. T. Hill: Phys. Rev. D 24 (1981) 691;

C. T. Hill, C. N. Leung, S. A. Rao: Nucl. Phys. B 262 (1985) 517;

W. A. Bardeen, C. T. Hill, M. Lindner: Phys. Rev. D 41 (1990) 1647.

[22] S. Dimopoulos, S. Theodorakis: Phys. Lett. B 154 (1985) 153. 


\section{Tables}

1. Left-handed fermions comprising an $S U(5)$ "quark" generation. The electric charges are in units of $\frac{1}{5}$ due to the charge quantisation rule.

2. Infrared fixed point Yukawa couplings and corresponding pole masses (for $F_{\pi}=75 \mathrm{GeV}$ ) for a particular choice of Yukawa couplings at the Planck scale.

\section{Figures}

1. $\alpha^{-1}$ from $M_{Z}$ to the Planck scale for each group. There are obviously no Landau poles so this model is self-consistent.

2. An example of running Yukawa couplings for all fermions with a mass the same order of magnitude as the electroweak scale. The values were chosen at the Planck scale and run down to $M_{Z}$ so that all the fermions would have a mass allowed by current experimental limits. 
Table 1:

\begin{tabular}{|c|c|c|}
\hline $\begin{array}{c}\text { Representation under } \\
S U(2) \otimes S U(3) \otimes S U(5)\end{array}$ & $\begin{array}{c}U(1) \text { Representation } \\
\frac{y}{2}\end{array}$ & $\begin{array}{c}\text { Electric Charge } \\
Q\end{array}$ \\
\hline $2,1,5$ & $-\frac{1}{10}$ & $\left(\begin{array}{c}\frac{2}{5} \\
-\frac{3}{5}\end{array}\right)$ \\
\hline $1,1, \overline{5}$ & $-\frac{4}{10}$ & $-\frac{2}{5}$ \\
\hline $1,1, \overline{5}$ & $\frac{6}{10}$ & $\frac{3}{5}$ \\
\hline
\end{tabular}


Table 2:

\begin{tabular}{|c|c|c|}
\hline Fermion & Yukawa Coupling at $M_{Z}$ & Pole Mass $(\mathrm{GeV})$ \\
\hline$y_{t}$ & 1.00 & 175 \\
\hline$y_{t^{\prime}}$ & 0.77 & 135 \\
\hline$y_{b^{\prime}}$ & 0.75 & 131 \\
\hline$y_{5 u}$ & 0.38 & 94 \\
\hline$y_{5 d}$ & 0.40 & 97 \\
\hline
\end{tabular}




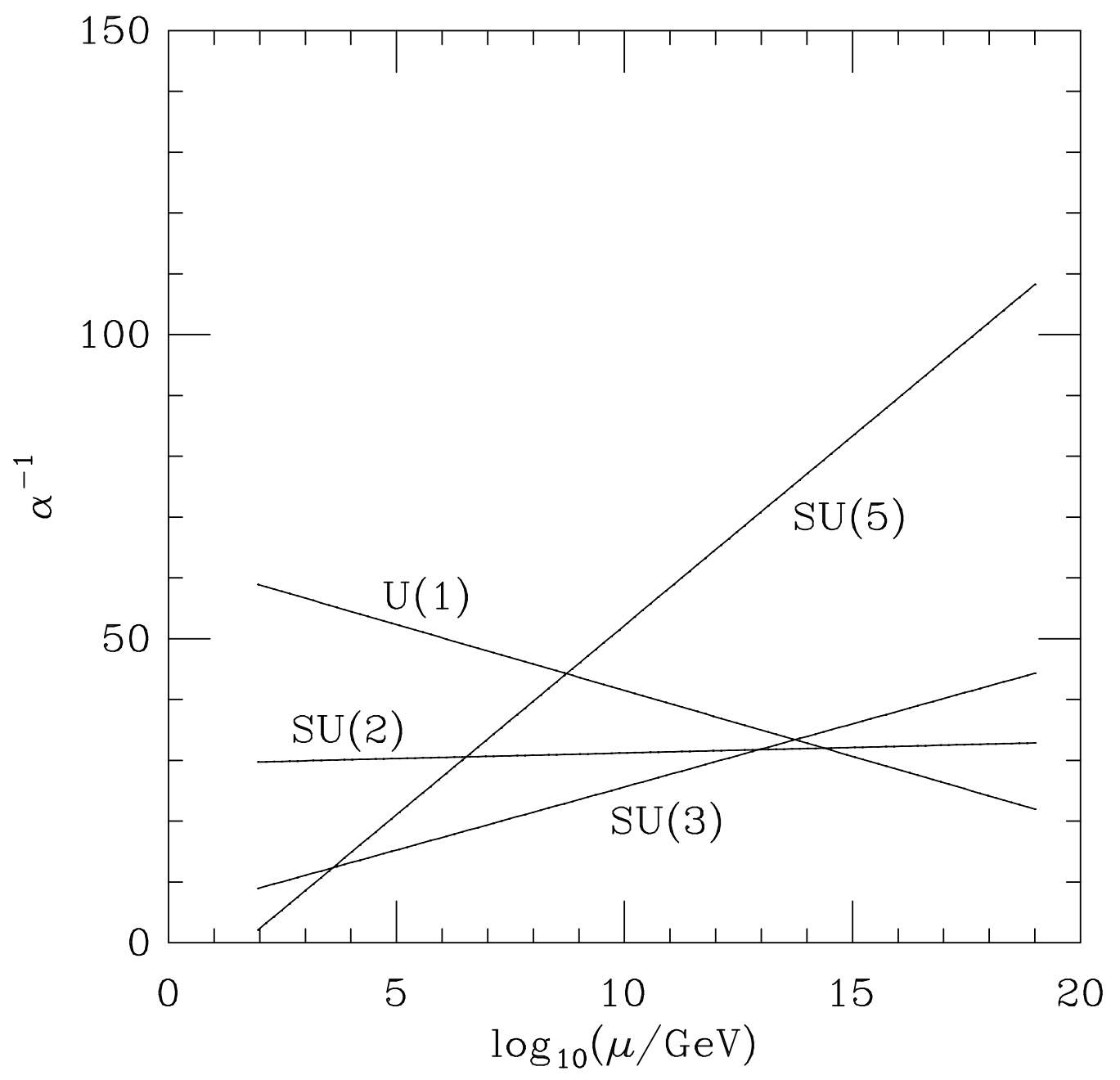

Figure 1: 


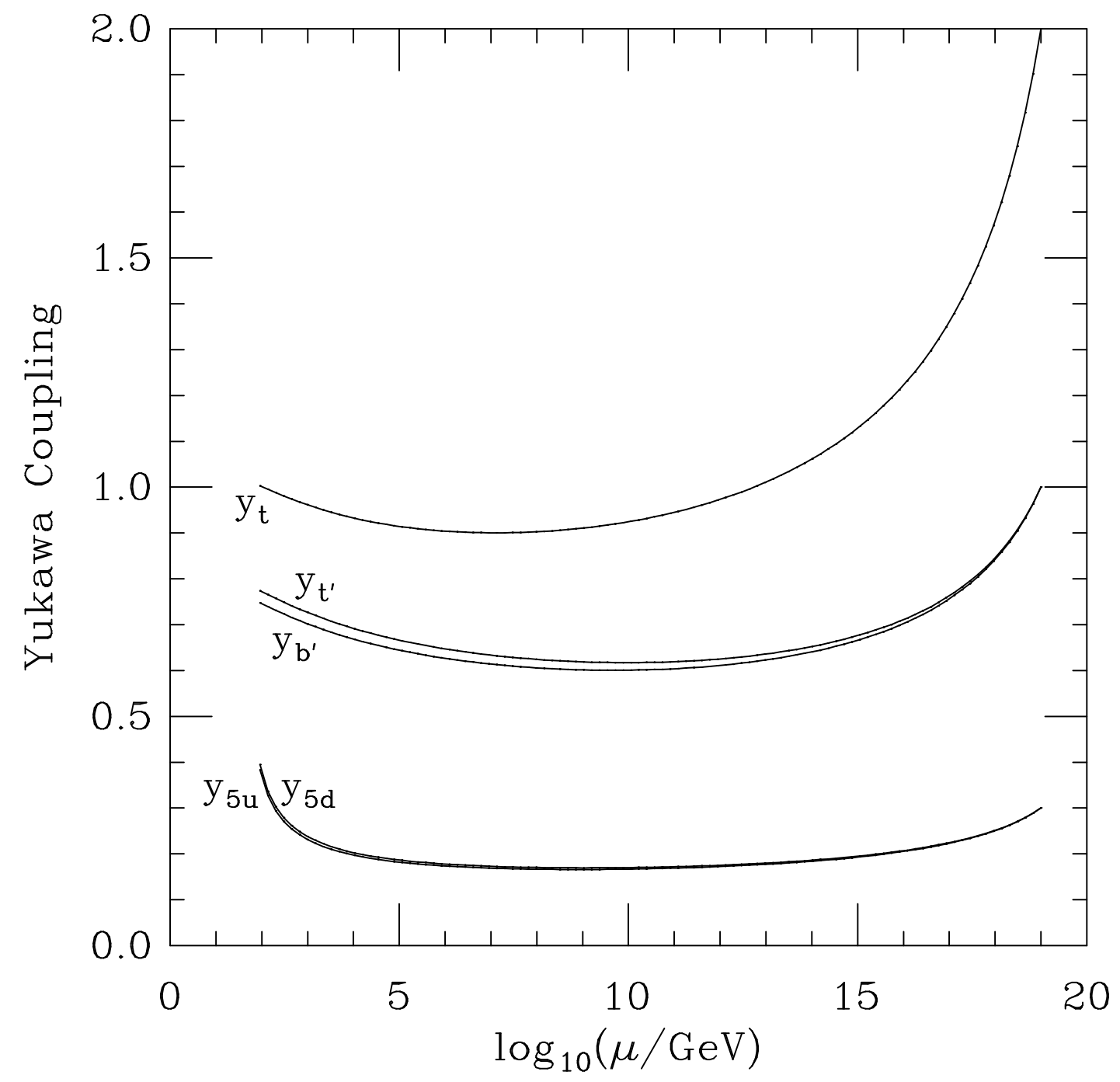

Figure 2: 